\title{
Estrés en la carrera de Ingeniería Civil
}

\section{Stress among civil engineering career}

\author{
Jennifer Espinosa ${ }^{1}$, Karla Ochoa $^{1,}$ María Saavedra ${ }^{1,}$ Carlos Rodríguez ${ }^{1,}$ Casilda Saavedra ${ }^{1 *}$ \\ ${ }^{1}$ Licenciatura en Ingeniería Civil - Facultad de Ingeniería Civil - Universidad Tecnológica de Panamá,
}

\begin{abstract}
Resumen Este estudio tuvo como objetivo identificar en qué año de la carrera de Ingeniería Civil se genera más estrés en los estudiantes universitarios. El estudio se realizó durante el primer semestre 2019 de la Universidad Tecnológica de Panamá. Para conocer en qué año se generó el mayor nivel de estrés se le aplicó una encuesta a una muestra representativa de estudiantes de cada año donde se analizó diferentes puntos relativos a reacciones físicas, psicológicas y cognitivas generadas por estrés, estresores y mecanismos de afrontamientos. Debido a los resultados encontrados, se concluyó que el año en el cual los estudiantes generan más estrés es el cuarto año de la carrera universitaria, destacándose diferentes estresores como, sobrecarga de tareas y trabajos, tiempo limitado para trabajos y la evaluación por parte de los profesores, y los mecanismos de afrontamiento más utilizados fueron la elaboración de un plan y ejecución de sus tareas, y la habilidad de asertividad.
\end{abstract}

Palabras clave Estrés, estresores, mecanismos de afrontamiento, salud mental, sueño, fatiga

Abstract This research aimed to identify wich year of the civil engineering career generates more stress in university students. The study was conducted during the first semester 2019 of the Technological University of Panama. In order to know, in what year the highest level of stress was generated, a survey was applied to a representative sample of students each year where different points related to physical, psychological and cognitive reactions, stressors and coping mechanisms were analyzed. Due to the results found it was concluded that the year in wich students generate more stress is the fourth year of the university degree, highlighting different stressors such as, overload of tasks and jobs, limited time for assignments and evaluation by teachers, and the most commonly used coping mechanisms were the elaboration of a plan and execution of their tasks, and the ability of assertiveness.

Keywords Stress, stressors, coping mechanisms, mental health, slumber, fatigue.

* Corresponding author: casilda.saavedra@utp.ac.pa

\section{Introducción}

Está comprobado que todos los organismos experimentan crisis de estrés durante su vida, pero cuando esto se prolonga produce fatiga en el individuo y genera una serie de problemas y situaciones [1]. Este es un tema que no se le ha dado la debida importancia y que afecta el rendimiento académico, rendimiento en el trabajo, la salud física y emocional del individuo, y que, genera consecuencias que resultan negativas en respuesta al estrés producido [2].

La salud mental abarca una amplia gama de actividades directa o indirectamente relacionadas con el componente de bienestar físico, mental y social y no solamente la ausencia de enfermedades.

El concepto de estrés se ha aplicado a diversos fenómenos psicosociales, lo que ha llevado a cierta confusión sobre el término. Sin embargo, puede considerarse como un estado producido por una serie de demandas inusuales o excesivas que amenazan el bienestar o integridad de una persona. Las investigaciones muestran que los problemas de salud mental son generados, activados o exacerbados ante la exposición de eventos estresantes [3]. Se puede afirmar que el estrés es la respuesta automática y normal del cuerpo humano ante situaciones que pueden resultar amenazadoras o atemorizantes [4].

La salud mental es tan importante como la salud física para el bienestar general de los individuos, las sociedades y los países. Pese a ello, solo una pequeña minoría de los 450 millones de personas que padecen un trastorno mental o del comportamiento está en tratamiento [5].

El comportamiento de una persona en relación con su propia salud depende en gran medida de su salud mental. Por ejemplo, datos recientes han demostrado que los jóvenes con trastornos psiquiátricos como la depresión y la dependencia de ciertas sustancias tienen más probabilidades de comenzar a fumar y de adoptar prácticas sexuales de riesgo.

Inferimos que los estudiantes universitarios se encuentran en un periodo de transición, donde la maduración de sus acciones y las medidas que adopten para enfrentarlas jugará un 
papel muy importante en su vida profesional. El cambio del colegio a la vida universitaria experimenta un alto nivel de estrés, vinculado a diferentes factores como lo son: la independencia de los padres, la elección de una carrera y el alto nivel de exigencia por parte de los profesores dentro de la universidad.

En la actualidad hay variedad de artículos de investigación y médicos que nos hablan de la problemática del estrés y todo lo que este involucra. Centrándonos en el estrés académico, cuando el estudiante estima que los requerimientos de una situación exceden sus recursos y capacidades, comienza a sentirse estresado. Si el desfase es muy acusado, sus pensamientos serán poco esperanzadores y sus emociones negativas, consecuentemente, su esfuerzo y su productividad disminuirán. En este caso, el estrés tiene un efecto negativo, constatado en la investigación sobre salud mental y física [6].

Si no se logra manejar el estrés en esta etapa de preparación y formación académica, podría afectar el conjunto de actividades a manejar para la vida laboral. De esta forma, el estrés laboral en Panamá, ya se está situado en la cabeza del ranking entre las enfermedades que agobian a la salud y bienestar de los panameños. Esto se traduce en importantes pérdidas económicas debido al mayor ausentismo y a la disminución de productividad [7].

Tomando en cuenta las situaciones de estrés en los estudiantes universitarios, este estudio pretende determinar el año con mayor estrés dentro de la carrera de ingeniería civil enfocado en el primer semestre del año 2019.

Con este estudio se pretende contribuir a disminuir esta problemática en la UTP al compartir los resultados con la Dirección de Orientación Psicológica de la Universidad Tecnológica de Panamá.

La hipótesis en nuestro estudio es que el año en que se presenta la mayor cantidad de estrés en la Universidad Tecnológica de Panamá para la carrera de ingeniería civil es el cuarto año.

Para probar la hipótesis, se tomó una muestra entre los estudiantes de los diferentes años de la carrera de ingeniería civil de la Universidad Tecnológica de Panamá del Campus Central Víctor Levi Sasso, y con esta muestra se realizó un análisis para identificar el año en el que se produce mayor estrés en los estudiantes durante el primer semestre del 2019.

El instrumento que se utilizó para validar esta información fue un cuestionario. La muestra se conformó por un total de 546 estudiantes cursantes de los distintos años de la carrera de Ingeniería Civil, correspondiente al primer semestre 2019 en la Universidad Tecnológica de Panamá. El muestreo realizado fue aleatorio simple. La muestra y el análisis fueron calculados a partir del paquete estadístico SPSS [8]. Se escogieron 28 ítems que pretendían predecir de manera confiable y asertiva el objetivo de esta investigación. Los ítems fueron presentados por los integrantes de este grupo de investigación en los diversos grupos de la carrera de ingeniería civil [9].

\section{Materiales y Métodos}

Este estudio es descriptivo, correlacional y transversal ya que el propósito de nuestra investigación es identificar en un momento dado, cuál es el año de la carrera de ingeniería civil donde se presenta el mayor estrés en los estudiantes.

\subsection{Sujetos}

Para este caso se decidió seleccionar muestras representativas aleatorias simples de cada año de estudio (Ver tabla 1). El modelo de investigación se conformó por un total de 546 estudiantes cursantes del primer semestre 2019, muestras obtenidas con el $90 \%$ de confiabilidad y un margen error permitido de $5 \%$, de la población de estudiantes debidamente matriculados de la carrera de ingeniería civil, campus Víctor Levi Sasso de la Universidad Tecnológica de Panamá. (Ver tabla 2).

Para generar la muestra por año de la carrera de ingeniería civil se empleó la ecuación 1.

En donde:

$$
n=\frac{N * Z^{2} * A * p * q}{(N-1) * E^{2}+Z^{2} * p * q}
$$

$n=$ Tamaño de muestra

$\mathrm{N}=$ Población

$\mathrm{Z}=$ Nivel de confianza

$p=\%$ de la poblacion que tiene el atributo deseado

$q=\%$ de la poblacion que no tiene el atributo deseado.

$\mathrm{E}=$ Error de estimación máximo permitido.

Para el cálculo de las muestras como no conocíamos antecedentes de este tipo de estudio dentro de la Universidad Tecnológica de Panamá, los valores de $p$ y $q$ se asumieron en un $50 \%$.

Tabla 1. Muestras obtenidas para el análisis de la investigación de población finita

\begin{tabular}{|c|c|}
\hline Año & Muestras \\
\hline I año & 64 \\
\hline II año & 73 \\
\hline III año & 110 \\
\hline IV año & 133 \\
\hline V año & 166 \\
\hline Total & 546 \\
\hline
\end{tabular}

Tabla 2. Cantidad de estudiantes matriculados en la carrera de licenciatura en ingeniería civil, período primer semestre 2019.

\begin{tabular}{|c|c|}
\hline Año & $\begin{array}{c}\text { Población Total de } \\
\text { estudiantes }\end{array}$ \\
\hline 1 & 83 \\
\hline 2 & 104 \\
\hline
\end{tabular}




\begin{tabular}{|c|c|}
\hline 3 & 184 \\
\hline 4 & 259 \\
\hline 5 & 431 \\
\hline Total & 1061 \\
\hline
\end{tabular}

Fuente: Dirección General de Tecnología de la Información y Comunicaciones.

\subsection{Instrumento}

El cuestionario se adoptó de la prueba SISCO del estrés académico, diseñado y validado por Barraza, el cual presenta altos niveles de confiabilidad [10].

El cuestionario está estructurado en 28 ítems divididos en tres bloques de información. El primer bloque (14 ítems) correspondió a reacciones o síntomas físicas, psicológicas y de comportamiento mental que los estudiantes presentan cuando estos muestran inseguridad o nerviosismo ante situaciones propias del ambiente universitario. El segundo bloque correspondió a estresores ( 8 ítems) el cual permitió identificar situaciones académicas dentro del entorno universitario que hacen al estudiante sienta inquietud. Por último, el tercer bloque concernió a estrategias de afrontamiento (seis times) estas fueron los modos en que los estudiantes enfrentan situaciones de estrés (ver Tabla 3).

El cuestionario poseía un modelo de selección múltiple tipo abanico [11], donde al encuestado se le presentó tres elecciones de acuerdo con la frecuencia (nunca, a veces y siempre), como indicadores que presentaban estos factores incidentes en el estrés académico.

Tabla 3. Estructura del cuestionario adoptado del SISCO del estrés académico

\begin{tabular}{|c|c|}
\hline \multirow{6}{*}{ Bloque 1} & Reacciones físicas \\
\hline & $\begin{array}{l}\text { Trastornos del sueño, fatigas crónicas, } \\
\text { dolores de cabeza, problemas de digestión, } \\
\text { rascarse o morderse las uñas }\end{array}$ \\
\hline & Reacciones psicológicas \\
\hline & $\begin{array}{l}\text { Inquietud, depresión o tristeza, ansiedad y } \\
\text { angustia, problemas de concentración, } \\
\text { agresividad e irritabilidad }\end{array}$ \\
\hline & Reacciones mentales \\
\hline & $\begin{array}{l}\text { Conflictos o tendencia que discutir, } \\
\text { aislamiento, desgano de labores académicas, } \\
\text { aumento o reducción de consumo de } \\
\text { alimentos. }\end{array}$ \\
\hline \multirow[b]{2}{*}{ Bloque 2} & Estresores \\
\hline & $\begin{array}{l}\text { Competencia, sobrecarga de tareas, } \\
\text { personalidad del profesor, evaluaciones, tipos } \\
\text { de trabajos, no comprender temas, } \\
\text { participación en clases, tiempo limitado. }\end{array}$ \\
\hline Bloque 3 & Estrategias de afrontamiento \\
\hline
\end{tabular}

\begin{tabular}{|l|l|}
\hline & $\begin{array}{l}\text { Asertividad, elaboración de planes, elogios, } \\
\text { religiosidad, búsqueda de información, } \\
\text { ventilación y confidencias. }\end{array}$ \\
\hline
\end{tabular}

\subsection{Recolección y análisis de los datos}

Se realizó una validación del cuestionario una vez efectuadas las recomendaciones de los asesores. La misma se aplicó a una muestra pequeña de 30 estudiantes, donde se evaluó la comprensión de la pregunta además del tiempo que se tomó para completar el cuestionario.

La aplicación del cuestionario se realizó con el debido permiso por parte del Decano de la Facultad de Ingeniería Civil de la Universidad Tecnológica de Panamá. La obtención de datos de las encuestas se realizó en el aula de clase y en horario académico, de forma voluntaria y anónima.

Para el análisis de los datos el procesamiento se tabuló y aplicó la estadística descriptiva inferencial mediante el programa estadístico IBM ${ }^{\circledR}$ SPSS ${ }^{\circledR}$ Statistics Versión 25 . La evaluación del cuestionario se basó en porcentajes de análisis estadístico como la media, moda, frecuencias absolutas y relativas de cada año de estudio.

En vista de que estábamos midiendo el nivel de estrés y la respuesta "siempre" es la que indica que los estudiantes están en presencia de estrés, el porcentaje se basó en los que dieron respuesta "siempre".

Para complementar el análisis se presentaron gráficas que formaron una visión más amplia y certera de la información obtenida.

\section{Resultados y discusión}

De la muestra total de la población, el 56.2\% representó al género masculino, el $43.6 \%$ al género femenino y un $0.2 \%$ no quiso seleccionar una opción.

Los rangos de edades fueron los siguientes: entre 18-23 años figuró un 79.5 \%, entre 24-30 años un 19.4\%, entre 31-40 años un $0.7 \%$ y entre $41-50$ años un $0.4 \%$.

En la investigación, la participación por año de estudio fue la siguiente: primer año representó un $11.7 \%$, segundo año un $13.4 \%$, tercer año un $20.1 \%$, cuarto año un $24.4 \%$ y quinto año un $30.4 \%$.

El cuestionario fue evaluado por bloque. A continuación, se muestran los resultados correspondientes a los porcentajes de la frecuencia a cada año de estudio.

\subsection{Bloque 1: Síntomas o Reacciones}

De acuerdo con los resultados obtenidos en este bloque, el trastorno del sueño obtuvo presencia en cuatro de los cinco años de estudio evidenciando así ser la reacción física que se manifiesta en la mayoría de los años con una frecuencia promedio de presentarse siempre de un $30 \%$. 
Se observa que cuarto y quinto año presentan una reacción física adicional, fatiga crónica en segundo lugar y dolores de cabeza o migraña en tercer lugar de las reacciones físicas más relevantes. Observando que los estudiantes de cuarto año presentan fatiga crónica y los de quinto año dolores de cabeza, siendo factores que no se presentan en ninguno de los otros años. El tercer año no presentó ninguna reacción física considerable (ver figura1).

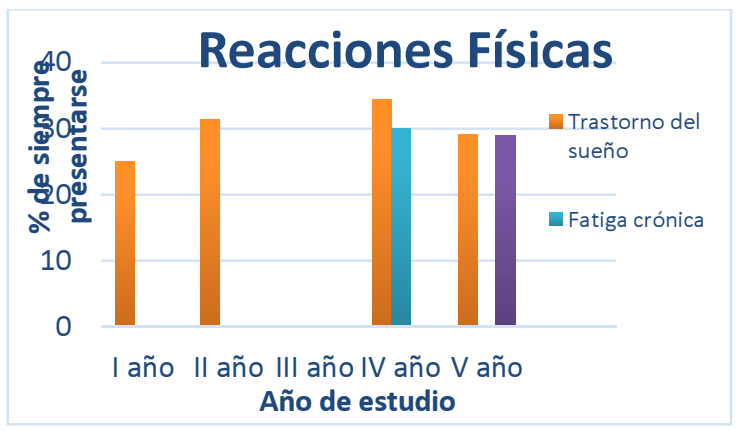

Figura 1. Reacciones físicas más frecuentes en los estudiantes.

De las reacciones psicológicas, la inquietud mantuvo presencia en cuatro de los cinco años de estudio (ver figura 1); esta debe ser la reacción psicológica con frecuencia presentada por los estudiantes con un promedio de $29 \%$.

Se observa que el año que presentó una mayor cantidad de reacciones psicológicas fue el cuarto año con cuatro reacciones psicológicas producto del estrés. También se observó que en primer año ninguna de las reacciones psicológicas presentadas demostró ser frecuente en ese año de estudio (ver figura 2).

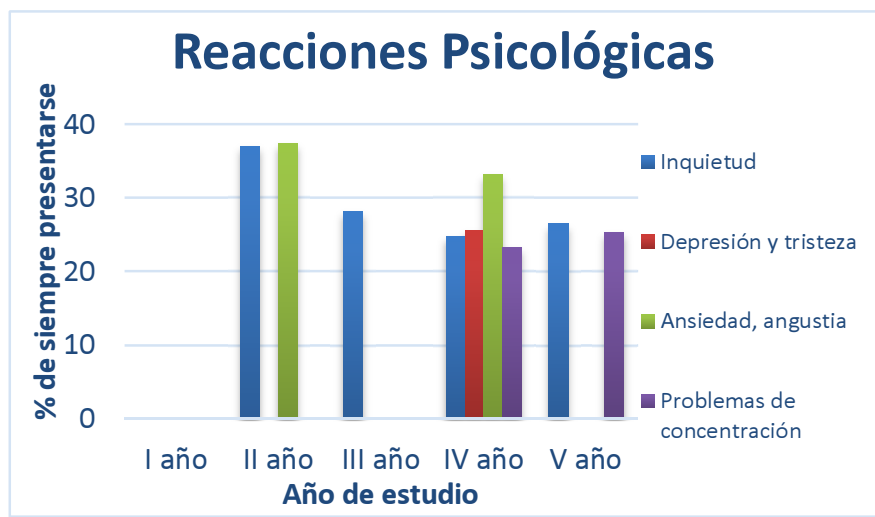

Figura 2. Reacciones psicológicas más frecuentes en los estudiantes.

De las reacciones conductuales, el desgano por las labores universitarias mostró ser la reacción más presentada por los estudiantes siempre cuando éstos tienen episodios de nerviosismo, reflejándose en cuatro años donde se estudió presentando un promedio de siempre presentarse de un $26 \%$.

Se observa que, el año con la mayor cantidad de reacciones conductuales fue cuarto año. Por ende, este es el salón que genera más respuestas de estrés con respecto a las reacciones conductuales. Además, se observó que el primer año no presentó ninguna reacción psicológica frecuente ante el estrés (ver figura 3 ).

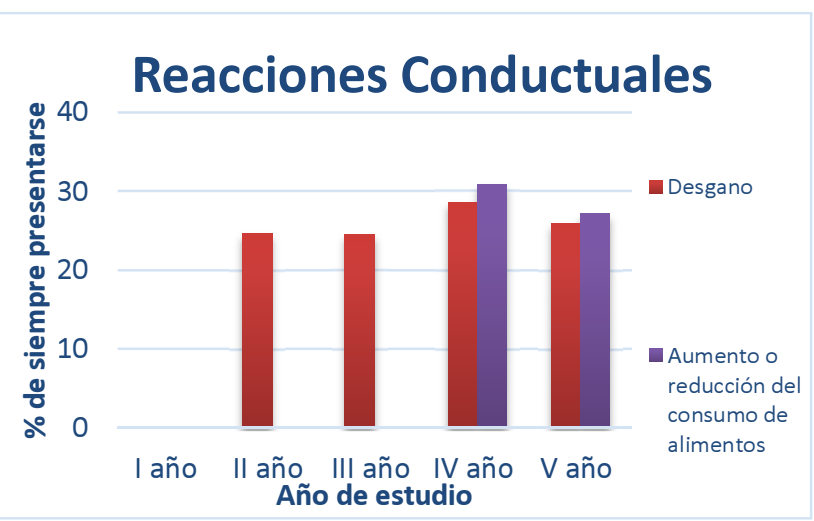

Figura 3. Reacciones conductuales más frecuentes en los estudiantes.

Los porcentajes mostrados corresponden a la afirmación de que los estudiantes siempre presentaban estas reacciones ante situaciones de nerviosismo o situaciones propias de la universidad.

\subsection{Boque 2: Estresores}

Los resultados correspondientes de los estresores se encuentran en la figura 4. La sobrecarga de tareas y trabajos se reflejó como el principal estresor académico presentado en todos los años de estudio con un promedio de $46 \%$ de siempre inquietar al estudiante, seguido por, el tiempo limitado para trabajos y tercero, la evaluación por parte de los profesores.

Se observó que, tanto cuarto como quinto año presentaron seis estresores con más porcentajes de "siempre presentarse", pero las proporciones de estos estresores en cuarto año demostraron ser mayores que en quinto. (ver figura 4).

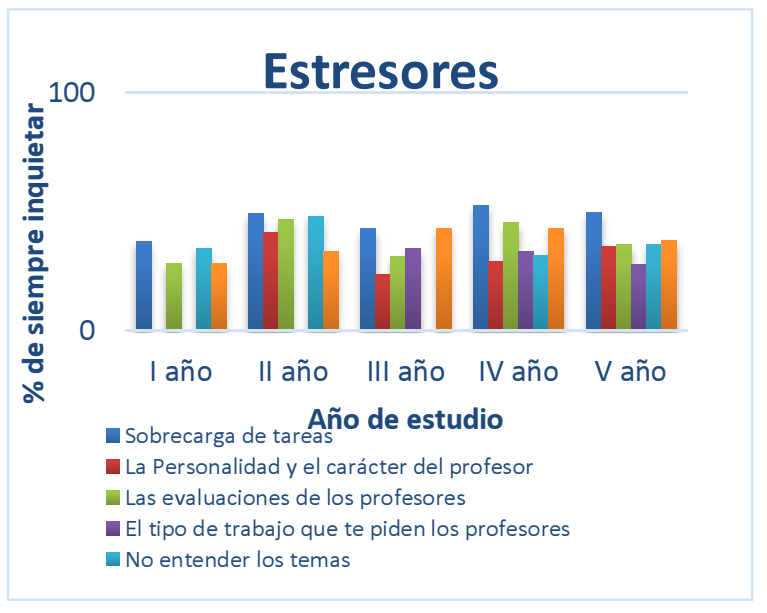

Figura 4. Estresores más frecuentes en los estudiantes. 


\subsection{Bloque 3: Estrategias de afrontamiento}

Los resultados correspondientes a las estrategias de afrontamiento se muestran en la tabla 4. La elaboración de un plan y ejecución de sus tareas se presenta como la principal estrategia empleada por los estudiantes en cuatro de los cinco años de estudio, con un promedio de $37 \%$ de "siempre emplearse", seguido por la habilidad de asertividad.

Se observó que, primero y segundo año presentan cuatro estrategias de afrontamiento, pero los porcentajes en primer año son más altos en comparación con segundo año; lo que demuestra que los estudiantes de primer año manejan parte de sus reacciones empleando estrategias que ayudan a disminuir los estresores y no evidencian reacciones de estrés (figura 5).

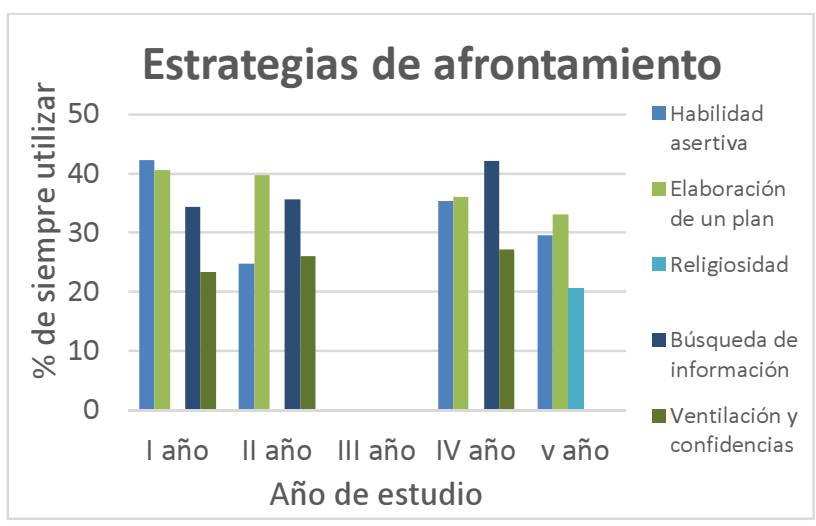

Figura 5. Estrategias de afrontamiento más frecuentes en los estudiantes.

\section{Conclusiones}

El objetivo de este proyecto fue determinar el año con mayor estrés dentro de la carrera de ingeniería civil durante el primer semestre 2019, demostrando qué factores de estrés son más recurrentes al estudiante y generadores de éste.

Mediante este estudio se comprueba la hipótesis de que el cuarto año de la carrera de ingeniería civil en la Universidad Tecnológica de Panamá es el año en el que los estudiantes presentan un nivel de estrés mayor al de los demás años de la carrera.

Con los resultados obtenidos, se puede demostrar que, entre las reacciones físicas, psicológicas y comportamientos más recurrentes como trastornos del sueño, inquietud, problemas de concentración y desgano para realizar las labores universitarias, sobrecarga de tareas, evaluaciones de los profesores, el tipo de trabajo de piden, no entender el tema dado, participación en clase, tiempo limitado de entrega son los factores más recurrentes en nuestro estudio, en donde se demuestra que los porcentajes (\%) más altos recae en el cuarto año de estudio.

Por otro lado, también recurren a las siguientes estrategias ante el problema, como la habilidad asertiva, elaboraciones de planes y ejecución de las tareas y búsqueda de información sobre la situación, es por esto por lo que recomendamos al
Departamento de Orientación Psicológica para un mejor rendimiento del estudiante técnicas de estudio, charlas motivacionales, que ayuden a los estudiantes a minimizar estos factores y no sea esto motivo de abonar la carrera.

Consideramos, además, que el Departamento de Bienestar Estudiantil y Orientación Psicológica deben trabajar de la mano, brindando apoyo continuamente al estudiante.

\section{AGRADECIMIENTO}

Agradecemos a la Lic. Artemia A. Mojica de la Dirección de Orientación Psicológica, por su asistencia en el área de la psicología y por los conocimientos obtenidos durante esta investigación. También agradecemos a la Dra. Casilda Saavedra de Madrid por su compromiso y asistencia al aportar los conocimientos que ayudaron a esta investigación.

\section{REFERENCIAS}

[1] Marco Antonio Pulido Rull, María Luisa Serrano Sánchez, Estefanía Valdés Cano, María Teresa Chávez Méndez, Pamela Hidalgo Montiel y Fernando Vera García. Estrés Académico en Estudiantes Universitarios, artículo Psicología y Salud, Vol. 21, Núm. 1: 31-37, enero-junio de 2011.

[2] Lya Feldman, Lila Goncalves, Grace Chacón-Puignau, Joanmir Zaragoza, Nuri Bagés, Joan de Pablo. Relaciones entre Estrés Académico, Apoyo Social, Salud Mental y Rendimiento Académico en Estudiantes Universitarios Venezolanos. Artículo - Universidad Simón Bolívar, Caracas, Venezuela.

[3] Mónica Cassaretto, Cecilia Chau, Haydeé Oblitas, Nancy Valdez. Estrés y Afrontamiento en Estudiantes de Psicología. Artículo - Revista de Psicología de la PUCP. Vol. XXI, 2, 2003.

[4] Barrios, Carroll, Norvin Steven Frías(Mediadero). El Estrés Laboral Visión Gerencial, Trabajo De Grado De Gerencia En Servicios De Salud En La Universidad Metropolitana De Educación, Ciencias Y Tecnología.

[5] Informe sobre la salud en el mundo, OMS, Organización Mundial de la Salud 2001.

[6] Rafael García-Ros, Francisco Pérez-González, Josefa Pérez-Blasco, Luis A. Natividad. Evaluación del Estrés Académico en Estudiantes de Nueva Incorporación a la Universidad. Artículo - Universidad de Valencia 2010.

[7] María Olvido Martín Graciani, Francisco David Trujillo Aguilera, Noelia Moreno Morales. Estudio y Evaluación del estrés en estudiantes de grado de Ingeniería Industrial en la Universidad de Málaga. Artículo - Revista educativa Hekademos, 13, junio 2013.

[8] Carlos Alberto Román Collazo, Felino Ortiz Rodríguez, Yenima Hernández Rodríguez. El Estrés Académico en 
Estudiantes Latinoamericanos de la Carrera de Medicina. Artículo - Escuela Latinoamericana de Medicina, Cuba.

[9] Ramón G. Cabanach, Antonio Valle, Susana Rodríguez, Isabel Piñeiro, y Carlos Freire. Escala de Afrontamiento del Estrés Académico (A-CEA). Artículo - Revista Iberoamericana de Psicología y Salud, 2010, 1(1): 51-64.
[10]Barraza, Macías A, Propiedades psicométricas del inventario SISCO del estrés académico, revista electrónica psicología científica febrero 262007

[11] R. Sierra Bravo "Técnicas de Investigación Social", Madrid Paraninfo, 1994. 